\begin{tabular}{|c|l|}
\hline Title & Fluid Permeability in Stratified Unconsolidated Particulate Bed \\
\hline Author(s) & Otomo, Ryoko; Harada, Shusaku \\
\hline Citation & $\begin{array}{l}\text { Transport in Porous Media, 96(3), 439-456 } \\
\text { https://doi.org/40.1007/311242-012-0098-6 }\end{array}$ \\
\hline Issue Date & 2013-02 \\
\hline Doc URL & http://hdl.handle.net/2115/52562 \\
\hline Type & article (author version) \\
\hline File Information & TIPM.96(3).439-456.pdf \\
\hline
\end{tabular}

Instructions for use 


\title{
Fluid Permeability in Stratified Unconsolidated Particulate Bed
}

\author{
Ryoko Otomo · Shusaku Harada \\ R. Otomo \\ Research Fellow of the Japan Society for the Promotion of Science \\ Faculty of Engineering, Hokkaido University, N13-W8, Sapporo, Hokkaido, 060-8628, Japan \\ Tel: +81 117066311 \\ Fax: +81 117066311 . \\ e-mail: ootomo@trans-er.eng.hokudai.ac.jp
}

\author{
S. Harada \\ Faculty of Engineering, Hokkaido University, N13-W8, Sapporo, Hokkaido, 060-8628, Japan \\ e-mail: harada@eng.hokudai.ac.jp
}

\begin{abstract}
Fluid permeability of polydisperse particulate bed with finite thickness has been examined. On the assumption of creeping flow, the permeability of monodisperse particles with arbitrary arrangement is calculated by means of Stokesian dynamics approach in which the interaction between individual particles and interstitial fluid is described by multipole expansion of the Oseen tensor. We have extended such calculation method to polydisperse particulate systems which have not so dense structures (up to particle volume fraction $\phi \sim 0.2$ ). The particles are located infinitely in space and their interaction has been taken into account by Ewald summation technique. For the spatial distribution of polydisperse particles, we consider locally-stratified particulate beds and define stratification degree as a parameter which apparently and mathematically represents the thickness of the mixing region of different-sized particles. The permeability profiles in the particulate beds with different stratification degree show the dependence of local permeability on the spatial and size distribution of particles. Consequently, the calculation results indicate that the permeability of non-uniform polydisperse particulate bed can be predicted by integrating the local permeation resistance which is determined by the local specific surface area.
\end{abstract}

\section{Keywords:}

Permeability, Porous media, Stokes flow, Polydisperse particles, End effect

\section{Introduction}

Fluid permeation through porous media is closely related to various engineering processes such as filtration and separation of the substances (Innocentini et al. 2009; Liekens et al. 2011), paper coating (Vidal et al. 2009), trickle-bed reaction, and radionuclide isolation (Hirono et al. 2003). For better performance and higher efficiency of these processes, it is important to evaluate properly the permeation characteristics which depend on the pore structures of the media. 
In general, the permeation characteristics are evaluated according to Darcy's law which relates the velocity of permeation flow to the pressure gradient. The flowability in the media is expressed as the permeability. For theoretical description of the permeability, the porous media is approximately expressed by assemblies of simple-shaped materials such as spherical particles or cylinders. Kozeny-Carman equation for low Reynolds number and Ergun equation for finite Reynolds number are well-known and commonly used for predicting the permeability of particulate bed. In these equations, the permeability is expressed as functions of particle properties such as diameter and volume fraction. On the other hand, cylindrical assemblies are used for modeling of fibrous materials (Jackson and James 1986). Filtration resistance of fibrous media has been predicted from the drag force acting on arrays of cylinders (Clague and Phillips 1997; Drummond and Tahir 1984; Sangani and Acrivos 1982).

The permeability in particulate media is essentially determined by the resistivity of constituent particles. Since the drag force acting on individual particles varies with their positional relationship, the microstructure of the particulate media is important for understanding the permeability. The drag force acting on arrays of particles in Stokes flow have been studied since early times. When the particle volume fraction is small, the permeability can be obtained by adding the resistivity of individual particles (Happel and Brenner 1991). On the other hand, the complicated hydrodynamic interaction becomes influential for larger particle volume fraction and in consequence the permeability cannot be described in a simple manner any more (Brinkman 1947; Happel 1958). Such complicated systems have been studied by direct computational simulation by Lattice Boltzmann method (LBM) (Hill et al. 2011a, 2011b) and analytical solution of Stokes equation (Ladd 1990).

In addition to the microstructures, the presence of ends also causes anisotropy in the structure and consequently it affects the permeability. The fluid-particle interaction in semiinfinite particle arrays in which particles occupy a half-space has been investigated by Ishii (1979) and Sangani and Behl (1989). They have reported that the drag force acting on monodisperse particles located in the vicinity of the ends is different from that on the inner particles. We have also investigated the end effect of finite particulate bed on the fluid permeability and have found the dependency of the end effect on particle radius in the previous work (Otomo and Harada 2011). 
The polydispersity of particles also makes the prediction of the permeability complicated. Koo and Sangani (2002) have solved Stokes equation in the presence of bidisperse particles and have predicted the hydrodynamic transport properties. van der Hoef et al. (2005) have derived the analytical expression of the drag force on bidisperse spheres in Stokes flow by fitting the simulation results of LBM. Sarkar et al. (2009) have verified that the expression by van der Hoef et al. (2005) is applicable to polydisperse particulate beds in which the ratio between the smallest and the largest particles is lower than 1:5. For the estimation of the permeability of highly polydisperse systems, Vidal et al. (2009) derived the modified KozenyCarman equation by describing the Kozeny constant depending on the particle size distribution. Based on the simulation and experimental data of previous studies, Cello et al. (2010) have proposed a revised model in computationally-efficient form.

The above studies are valuable for the prediction of the permeation characteristics in fully-mixed polydisperse bed. However, actual permeation media have occasionally heterogeneous structures, namely they have locally-stratified structures such as geological layer, filtration tank and so on. The non-uniform structures and the end effect in polydisperse particulate bed have not been considered in the evaluation of the permeability. In this study, we consider Stokes flow through stratified polysidperse particulate bed with finite bed thickness. Figure 1 shows the illustration of particulate beds with uniform, completely-layered, and intermediate structures. As the expressions of the permeability for uniform and layered structures are to be found, the permeability for intermediate structures is particularly investigated in this article. In the next section, we briefly introduce some important equations and definitions. In section 3, our calculation procedure by the multipole expansion of the Oseen tensor and the application of two-dimensional periodic boundary are shown. In section 4, the results are shown in three stages. First, we validate our results for uniform particulate bed by comparison with those by previous studies. Second, effects of stratification inside bidisperse particulate bed on the permeability are discussed. Then, we extend the discussion to the polydisperse system. Finally, we conclude in section 5 . 


\section{Background}

As a fundamental knowledge to investigate the permeability of the heterogeneous particulate bed shown in Fig. 1, we introduce some of the existing equations. The definition of fluid permeability in low Reynolds flow is given by Darcy's law,

$$
u=k \frac{\Delta P}{\mu L},
$$

where $\mu$ is fluid viscosity, $u$ is superficial fluid velocity, $\Delta P / L$ is pressure gradient, and $k$ is permeability with dimension of square of the length. It is known that the permeability $k$ depends on porosity and porous structure inside the media. If the porous structure is uniformly at random, the permeability of monodisperse particulate bed is described as a function of particle radius $a_{0}$ and particle volume fraction $\phi$ as

$$
k_{\text {mono }}=k_{\text {mono }}^{*}(\phi) a_{0}{ }^{2},
$$

where $k_{\text {mono }}^{*}(\phi)$ is the dimensionless permeability. $k_{\text {mono }}^{*}(\phi)$ can be derived from well-known Kozeny-Carman equation (Carman 1937) and is

$$
k_{\text {mono }}^{*}(\phi)=\frac{(1-\phi)^{3}}{45 \phi^{2}} .
$$

The above equation is valid for dense conditions (large particle volume fraction). On the other hand, Brinkman has studied the drag force acting on particle assembly and has derived the permeability for dilute conditions which is called Brinkman equation (Brinkman 1947):

$$
k_{\text {mono }}^{*}(\phi)=\frac{2}{9 \phi}\left(1+\frac{3}{4} \phi\left(1-\sqrt{\frac{8}{\phi}-3}\right)\right) .
$$

In studies of recent years, van der Hoef et al. (2005) have proposed the expression of permeability from dilute conditions $(\phi \sim 0.01)$ to random closest packing condition ( $\phi=0.64)$. They have formulated the relation between particle volume fraction $\phi$ and the drag force acting on monodisperse particles, which is in extremely good agreement with data from direct numerical simulation by LBM. From this relation, the permeability results in

$$
k_{\text {mono }}^{*}(\phi)=4\left(180 \frac{\phi^{2}}{(1-\phi)^{3}}+18 \phi(1-\phi)(1+1.5 \sqrt{\phi})\right)^{-1} .
$$

On the basis of the monodisperse case, van der Hoef et al. (2005) and Sarkar et al. (2009) 
have derived the permeability for bi- and polydisperse particles. Prior to the introduction of their results, the important parameters are defined in a similar way to their articles. $N_{l}$ particles of radius $a_{l}$ are considered in which $l$ represents the particle species with $l=1,2, \cdots$, c. $c$ is the number of different kinds of particles. The mass fraction and the diameter fraction are defined respectively as

$$
x_{l}=\frac{\phi_{l}}{\phi}, y_{l}=\frac{a_{l}}{\langle a\rangle},
$$

where $\phi_{l}$ is the volume fraction of particle $l$, and $\phi_{l}=\left(4 \pi a_{l}{ }^{3} N_{l} / 3\right) / V_{\text {tot }} . V_{\text {tot }}$ is the total volume of the system. $\langle a\rangle$ is area-averaged radius (a half of Sauter diameter) which is defined by

$$
\langle a\rangle=\frac{\sum_{l=1}^{c} N_{l} a_{l}^{3}}{\sum_{l=1}^{c} N_{l} a_{l}^{2}}=\left[\sum_{l=1}^{c} \frac{x_{l}}{a_{l}}\right]^{-1}=a_{0}\left[\sum_{l=1}^{c} \frac{x_{l}}{R_{l}}\right]^{-1},
$$

where $R_{l}=a_{l} / a_{0}$ indicates the radius ratio and $a_{0}$ is the representative radius and generally one of $a_{l}$ is selected as $a_{0}$. For bidisperse particulate bed with the radii $a_{1}$ and $a_{2}$, dimensionless area-averaged radius results in

$$
\frac{\langle a\rangle}{a_{2}}=\frac{R}{x_{1}+R x_{2}},
$$

where the representative radius is set to be $a_{2}$ and $R$ represents $a_{1} / a_{2}$. van der Hoef et al. (2005) and Sarkar et al. (2009) described dimensionless permeability of polydisperse arrays of particles as follows (A typographical error in the article is modified.):

$$
k^{*}=k_{\text {mono }}^{*}(\phi) \frac{\langle a\rangle^{2}}{a_{0}^{2}}\left(1+0.064(1-\phi) \sum_{l=1}^{c} x_{l} y_{l}\right)^{-1} .
$$

In Eq. (9), the permeability depends not only on the particle volume fraction but also on the particle size distribution by which $x_{l}, y_{l}$, and $\langle a\rangle$ are determined. Equation (9) is a description of the permeability for uniform (fully-mixed) particulate bed shown in Fig.1c.

On the other hand, if particulate bed is completely stratified and the constituent layers can be assumed as monodisperse ones with different radius (see Fig.1a), the permeability can be obtained from adding the permeation resistance of each monodisperse layer as follows:

$$
k^{*}=\frac{1}{a_{0}^{2}}\left[\sum_{l=1}^{c} \frac{L_{l}}{L} \frac{1}{k_{l}}\right]^{-1}=\left[\sum_{l=1}^{c} \frac{L_{l}}{L} \frac{1}{k_{l} / a_{0}^{2}}\right]^{-1},
$$


where $L$ and $L_{l}$ are the thickness of total particulate bed and constituent layers respectively (see Fig.1a). Note that $k_{l}$ is the permeability of particulate bed with radius $a_{l}$ and particle volume fraction $\phi_{l}$, and it follows that $k_{l}=k_{\text {mono }}^{*}\left(\phi_{l}\right) a_{l}^{2}$ from Eq. (2). Accordingly, Eq. (10) can be written by

$$
k^{*}=\left[\sum_{l=1}^{c} \frac{L_{l}}{L} \frac{1}{k_{\mathrm{mono}}^{*}\left(\phi_{l}\right) a_{l}^{2} / a_{0}^{2}}\right]^{-1}=\left[\sum_{l=1}^{c} \frac{L_{l}^{*}}{L^{*}} \frac{1}{k_{\mathrm{mono}}^{*}\left(\phi_{l}\right) R_{l}^{2}}\right]^{-1},
$$

where $L^{*}$ and $L_{l}{ }^{*}$ are the normalized thickness by the representative radius $a_{0}$.

To summarize, we can calculate the permeability of polydisperse particulate bed in two extreme cases shown in Fig.1. The permeability of uniform (fully-mixed) bed (Fig.1c) can be calculated by Eq. (9), while the permeability of completely-stratified bed (Fig.1a) can be calculated by Eq. (11). However, there have been no expressions for intermediate case (heterogeneous structure) shown in Fig.1b. We discuss the effect of internal stratified structures of the polydispersed bed on fluid permeability in later sections and propose a model which relates the permeability to spatial distribution of different-sized particles.

\section{Calculation Method}

The system where the particulate bed moves in a quiescent fluid with fixed relative position of particles is preliminarily considered. We have obtained in such a system the relation between particle velocity and drag force acting on each particle and converted it into the relation between fluid velocity and permeation resistance in fluid permeation by shifting the frame of reference. Consequently, fluid permeability in Stokes flow is calculated from this relation.

The relation between particle velocity and drag force acting on each particle has been obtained by Stokesian dynamics method proposed by Brady et al. (1988). Different-sized spherical particles move in a quiescent fluid with their relative position fixed (i.e. with constant velocity). The inertias of both particle and fluid are neglected on the assumption that the particles are adequately small and they moves slowly. The velocity of particle $\alpha$ is represented by $\boldsymbol{U}^{\alpha}$ and related to the force acting on each particle as follows: 


$$
6 \pi \mu a_{\alpha} \boldsymbol{U}^{\alpha}=\boldsymbol{F}^{\alpha}+\sum_{\substack{\beta=1 \\(\alpha \neq \beta)}}^{N} \boldsymbol{M}\left(\boldsymbol{x}^{\alpha}-\boldsymbol{x}^{\beta}\right) \cdot \boldsymbol{F}^{\beta},
$$

where $\mu$ is the fluid viscosity, $a_{\alpha, \beta}, \boldsymbol{x}^{\alpha, \beta}$, and $\boldsymbol{F}^{\alpha, \beta}$ are radius, position, and acting force of particles $\alpha, \beta$, respectively. The mobility matrix $M_{i j}(r)$ is calculated based on the Rotne-Prager tensor (Rotne and Prager 1969) which is derived from the multipole expansion of the Oseen tensor and Faxèn's law (Kim and Karrila 1991). For polydisperse particulate bed, $M_{i j}(\boldsymbol{r})$ is given by

$$
M_{i j}(\boldsymbol{r})=\left(\frac{3}{4} a_{\alpha}+\frac{1}{8} a_{\alpha}\left(a_{\alpha}{ }^{2}+a_{\beta}{ }^{2}\right) \nabla^{2}\right)\left(\nabla^{2} \delta_{i j}-\frac{\partial}{\partial x_{i}} \frac{\partial}{\partial x_{j}}\right) r .
$$

where $r=|\boldsymbol{r}|$ and $\boldsymbol{r}$ corresponds to $\boldsymbol{x}^{\alpha}-\boldsymbol{x}^{\beta}$, namely the distance of individual particles, for the calculation of Eq. (12). Note that Eq. (13) is obtained from the multipole expansion with zeroth-order term and the trace of second-order term on the assumption that the particle volume fraction is not so large. Ladd (1990) has analytically derived more accurate solution of Stokes equation in the presence of monodisperse particles at a wide range of particle volume fractions. However, as is explained below, it is extremely difficult to apply twodimensional Ewald summation (periodic boundary) to the mobility matrix with such higherorder terms. We focus attention on investigating of heterogeneous structural effects on the permeability and therefore calculate Eq. (13) on two-dimensional periodic boundary conditions. Consequently our analysis is restricted to the conditions that the particle volume fraction is not so large.

We calculate the permeability of particulate beds with three-dimensional $(x, y$ and $z$ direction) and two-dimensional ( $x$ and $y$ direction) periodic boundaries. The Ewald summation technique is applied in order to calculate properly the hydrodynamic effect of the further particles (Brady et al. 1988). For three-dimensionally infinite particulate bed, Ewald summation of Rotne-Prager tensor has been reported by Beenakker (1986). We calculate the permeability of the polydisperse system in a similar way as Beenakker by using Eq. (13).

For monodisperse particulate bed with two-dimensional periodic boundary, Ewald sum of Rotne-Prager tensor have reported by some recent works (Ichiki 1993; Harada and Otomo 2009; Otomo and Harada 2011) Two-dimensional Ewald summation technique for polydisperse bed is explained below. If we set the length of the calculation domain (the lattice 
width of Ewald sum) in $x, y$ directions to be $L_{1}, L_{2}$, respectively, the lattice vector is defined as $\boldsymbol{r}_{\gamma}=\left(n_{1} L_{1}, n_{2} L_{2}, 0\right)$ where $\left(n_{1}, n_{2}: 0, \pm 1, \pm 2 \ldots\right)$. The relation between the particle velocity and drag force acting on each particle is

$$
\begin{aligned}
6 \pi \mu a U_{i}^{\alpha}= & F_{i}^{\alpha}+\sum_{n_{1}, n_{2}} \sum_{\substack{\beta=1 \\
\beta \neq \alpha}}^{N} M_{i j}^{(1)}\left(\boldsymbol{x}^{\alpha}-\boldsymbol{x}^{\beta}+\boldsymbol{r}_{\gamma}\right) F_{j}^{\beta} \\
& +\frac{1}{L_{1} L_{2}} \sum_{\substack{\left.m_{1}, m_{2} \\
k \neq 0\right)}}^{N} \sum_{\beta=1}^{N} \exp \left[-i\left(k_{1}\left(x^{\alpha}-x^{\beta}\right)+k_{2}\left(y^{\alpha}-y^{\beta}\right)\right)\right] \tilde{M}_{i j}^{(2)}\left(k_{1}, k_{2} ; z^{\alpha}-z^{\beta}\right) F_{j}^{\beta} \\
& -M_{i j}^{(2)}(\boldsymbol{r}=0) F_{j}^{\alpha} .
\end{aligned}
$$

where $k_{1}=2 \pi m_{1} / L_{1}, k_{2}=2 \pi m_{2} / L_{2}\left(m_{1}, m_{2}: 0, \pm 1, \pm 2 \ldots\right)$. The mobility matrix for polydisperse particulate bed in real and reciprocal spaces $M_{i j}^{(1)}(\boldsymbol{r}), M_{i j}^{(2)}(\boldsymbol{r})$ and $\tilde{M}_{i j}^{(2)}\left(k_{1}, k_{2} ; z\right)$ are given as follows:

$$
\begin{gathered}
M_{i j}^{(1)}(\boldsymbol{r})=\left(\frac{3}{4} a_{\alpha}+\frac{1}{8} a_{\alpha}\left(a_{\alpha}{ }^{2}+a_{\beta}{ }^{2}\right) \nabla^{2}\right)\left(\nabla^{2} \delta_{i j}-\frac{\partial^{2}}{\partial r_{i} \partial r_{j}}\right)(\operatorname{rerfc}(\xi r)), \\
M_{i j}^{(2)}(\boldsymbol{r})=\left(\frac{3}{4} a_{\alpha}+\frac{1}{8} a_{\alpha}\left(a_{\alpha}{ }^{2}+a_{\beta}{ }^{2}\right) \nabla^{2}\right)\left(\nabla^{2} \delta_{i j}-\frac{\partial^{2}}{\partial r_{i} \partial r_{j}}\right)(\operatorname{rerf}(\xi r)), \\
\tilde{M}_{i j}^{(2)}\left(k_{1}, k_{2} ; z\right)=\int_{-\infty}^{\infty} \int_{-\infty}^{\infty} M_{i j}^{(2)}(r) \exp \left[i\left(k_{1} x+k_{2} y\right)\right] d x d y,
\end{gathered}
$$

where $\xi$ is the parameter for lattice sum. The lattice sum of real space shown as the second term in the right-hand side in Eq. (14) can be solved by the same procedure as the infinite Ewald sum. The lattice sum of reciprocal space shown as the third term is calculated by rewriting termwise the integrand of Eq. (17) and integrating each term. The numerical integration scheme is used for the calculation of a few terms in the above expressions.

Rewriting Eq. (14), we finally obtain the relation between the particle velocity and drag force acting on each particle in tensor form as follows:

$$
\boldsymbol{U}=\boldsymbol{M} \cdot \boldsymbol{F} .
$$

Equation (18) is known as the mobility equation of particles (Durlofsky et al. 1987; Jeffrey and Onishi 1984). We consider Eq. (18) as a solution of the resistance problem by transforming it to the inverse matrix

$$
\boldsymbol{F}=\boldsymbol{M}^{-1} \cdot \boldsymbol{U}
$$


When the particulate bed moves with given constant velocity $\boldsymbol{U}$, the drag force acting on them $\boldsymbol{F}$ can be calculated from Eq. (19). As mentioned above, we have converted this relation into the relation between fluid velocity and permeation resistance in fluid permeation through particulate bed which remains at rest. Note that $\boldsymbol{U}$ in Eq. (19) corresponds to superficial fluid velocity. On the basis of Darcy's law shown in Eq. (1), the permeability is calculated by the relation between $\boldsymbol{U}$ and $\boldsymbol{F}$.

\section{Results and discussion}

\subsection{Permeability in uniform bidisperse particulate bed}

The results of uniform particulate bed are exhibited for validation of our calculation method. We consider mono- and bidisperse particulate beds in which the constituent particles are arranged uniformly at random. Fig. 2 shows dimensionless permeability $k^{*}$ of threedimensionally infinite particulate bed for various particle volume fractions $\phi$. Our results for monodisperse particulate bed almost agree with those of previous studies which are mentioned in section 2. However, it is found that our results deviate from previous ones for larger $\phi$. This is because we truncate high-order terms in the multipole expansion as mentioned in section 3. Ladd (1990) has reported more accurate solution of Stokes equation in the presence of monodisperse particles which agrees well with the direct numerical simulation data in a wide range of particle volume fractions. While our results are less accurate than those by Ladd (1990), we can evaluate the effects of polydisperse and heterogeneous structures on the permeability. Afterward, we focus attention on the permeability of finite polydisperse particulate bed for $\phi=0.2$ in which the deviation is small enough not to affect the ensuing discussions.

Figure 2 also indicates the results for bidisperse particulate beds. $R$ represents the ratio of larger particle radius $a_{1}$ to smaller particle radius $a_{2}$, i.e., $R=a_{1} / a_{2}$. The calculations are performed for $R=2, R=3$, and $R=5$ under the condition that the mass fraction of particles with each radius are the same, i.e., $x_{1}=x_{2}=0.5$ in Eq. (6). Our results agree with what are calculated from Eq. (9) for $\phi \leq 0.2$. The results indicate that the permeability for larger $R$ is greater at 
constant $\phi$. This means that the presence of larger particles makes more connected interstices and consequently the fluid is easy to pass through the inside.

In order to validate the calculation method for finite particulate systems, the permeability of the finite (two-dimensionally infinite) bed is compared with that of the infinite bed. Figure 3 shows the permeability profiles inside the uniform particulate bed with finite thickness. The vertical axis represents the normalized position $z^{*}\left(=z / a_{0}\right)$ in flow direction. The horizontal axis represents the local permeability inside the bed relative to the permeability of infinite monodisperse bed which is shown in Fig. 2. The radius condition for the infinite monodisperse bed is $a / a_{0}=1.0$ and the volume fraction is $\phi=0.2$. As shown in Fig. 3, the results for monodisperse with $a / a_{0}=1.0$ indicate that the permeability represents unity at the center of the bed while it is greater in the vicinity of both ends. In other words, the permeability is the same as that of infinite particulate bed at the center of the bed and it varies at the ends. These variations of permeability are interpreted to be due to the anisotropic structure of the end. We have called them end effect in our previous work and have found that it ranges five to ten times as the constituent particle radius (Otomo and Harada 2011). In Fig. 3 , the end effect for monodisperse particulate bed with $a / a_{0}=2.0$ can be seen definitely compared with the condition of $a / a_{0}=1.0$. For bidisperse system which consists of the particles with both $a / a_{0}=1.0$ and 2.0 , the permeability is close to that for infinite bidisperse bed and the end effect show an intermediate tendency.

\subsection{Permeability in stratified bed of bidisperse particles}

In order to investigate the permeability of locally-stratified particulate bed, the partiallymixed bidisperse particulate bed is considered. The constituent larger and smaller particles (particle 1 and 2, respectively) have radii $a_{1}$ and $a_{2}\left(a_{1}>a_{2}\right)$. The representative length $a_{0}$ is set to be the smaller radius $a_{2}$ and the radius ratio is represented by $R=a_{1} / a_{2}$. Assuming that the mass fractions of each particle are $x_{1}=x_{2}=0.5$, we set the spatial distribution of particle 1 and 2 in $z$ direction (flow direction) by means of following volumetric existing probability $g_{1}\left(z^{*}\right)$ and $g_{2}\left(z^{*}\right)$, respectively, 


$$
\begin{gathered}
g_{1}\left(z^{*}\right)=\frac{1}{2}\left[1+\operatorname{erf}\left(-\frac{z^{*}}{\lambda}\right)\right], \\
g_{2}\left(z^{*}\right)=1-g_{1}\left(z^{*}\right)=\frac{1}{2}\left[1-\operatorname{erf}\left(-\frac{z^{*}}{\lambda}\right)\right],
\end{gathered}
$$

where $z^{*}\left(=z / a_{2}\right)$ is the normalized position and $z^{*}=0$ locates at the midpoint of the bed. $\lambda$ is a dimensionless parameter which represents "degree of stratification" of the particulate bed. From Eq. (20), the probability density function of particle 1 (larger particles) follows that $p_{1}\left(z^{*}\right)=g_{1}\left(z^{*}\right) /\left[a_{2} \int_{-L^{*} / 2}^{L^{*} / 2} g_{1}\left(z^{*}\right) d z^{*}\right]$. Figures 4 and 5 show the existing probability (Eq. (20)) of particle 1 (larger particles) and the illustration of the respective particulate beds. The dimensionless bed thickness $L^{*}$ is set to be 60 . The radius ratio and the particle volume fraction are $R=3$ and $\phi=0.2$, respectively. As shown in Figs. 4 and 5 , the bed for small $\lambda$ represents sharply-defined stratified structure while that for large $\lambda$ represents uniform (wellmixed) structure. In the intermediate cases, particles are mixed according to value of $\lambda$.

Here we consider the physical and mathematical meaning of parameter $\lambda$. The error function in Eqs. (20) and $(21)$ is $\operatorname{erf}(1) \approx 0.84$ for $z^{*}=-\lambda$ and $\operatorname{erf}(-1) \approx-0.84$ for $z^{*}=\lambda$. This means that the larger and the smaller particles are mixing at a rate of $0.92 \geq g_{1}\left(z^{*}\right) \geq 0.08$ and $0.08 \leq g_{2}\left(z^{*}\right) \leq 0.92$ for $-\lambda \leq z^{*} \leq \lambda$. Therefore, the length $2 \lambda$ is mathematically interpreted as the characteristic thickness of the mixing region. Such a definition of the mixing region is consistent with what one can apparently observe in Fig. 5.

Figure 6 shows the permeability profiles in the particulate beds which are shown in Figs. 4 and 5. The fluid passes toward positive $z$ direction (from the bottom to the upper side in Fig. 5). The permeability profile for large $\lambda(\lambda=1000)$ is almost the same as that for the uniform bidisperse bed shown in Fig. 3. On the other hand, the permeability for small $\lambda(\lambda=1)$ varies definitely at the midpoint of the bed because the particulate bed is close to bilayered structure. In this case, the permeability of each layer almost agrees with that for monodisperse bed with respective radii. For intermediate cases, the permeability profile changes continuously according to the existing probability of small and large particles. The end effect, which is shown in Fig.3, appears prominently at the bottom end of the bed for larger $\lambda$, where the large 
particle exists in large numbers.

Figure 7 shows the relation between the stratification degree $\lambda$ and the total permeability of the bidisperse bed. The permeability is normalized by the results of the monodisperse bed for the same volume fraction $(\phi=0.2)$ which is shown in Fig.2. It is found from Fig.7 that the total permeability depends on the stratification degree $\lambda$ and the dependence is greater in the case of larger radius ratio $R$. In all cases, the total permeability is small for small $\lambda$. This is because the particulate bed for small $\lambda$ has bilayered structures and the layer of smaller particles acts as a bottleneck of permeation flow.

As mentioned above, the length $2 \lambda$ means the characteristic thickness of mixing region. Therefore the particulate bed is mixed ambiguously when value of $\lambda$ is comparable to the bed thickness, i.e., from $\lambda=10$ to 100 in our conditions. As a result, the permeability under such conditions is greatly influenced by $\lambda$. As described in detail later, the above discussions by means of $\lambda$ would be valuable for a practical use on the estimation of permeation characteristics of heterogeneous media.

Here we consider the transition of the total permeability from bilayered bed (Fig.5a) to uniform (well-mixed) bidisperse bed (Fig.5d). As a limit of small $\lambda$, the total permeability of the completely-layered bidisperse bed can be calculated from Eq. (11). Equation (11) for bilayered particulate bed is written by

$$
k^{*}=\left(\frac{L_{1}^{*}}{L^{*}} \frac{1}{k_{\mathrm{mono}}^{*}\left(\phi_{1}\right)}+\frac{L_{2}^{*}}{L^{*}} \frac{1}{k_{\mathrm{mono}}^{*}\left(\phi_{2}\right) R^{2}}\right)^{-1} .
$$

If we set that the thickness of each layer is $L_{1}{ }^{*}=L_{2}{ }^{*}=0.5 L^{*}$ under the condition of mass fractions $x_{1}=x_{2}=0.5$ and that the particle volume fraction is constant in overall bed, Eq. (22) follows that $k^{*}=k_{\text {mono }}^{*}(\phi)\left[2 R^{2} /\left(1+R^{2}\right)\right]$. This equation is also shown in Fig.7 as a limit of small $\lambda$. It is found that the calculation results are in good agreement with this relation for small $\lambda$.

As a limit of large $\lambda$, Fig. 7 also shows the permeability of uniform bidisperse bed by van der Hoef et al. (2005) described in Eq. (9). It is found that our results almost agree with them. In addition, on the assumption that the second term in the parentheses in Eq. (9) is small, we 
also plot the approximation form of Eq. (9) for a large limit of $\lambda$ as

$$
k^{*}=k_{\text {mono }}^{*}(\phi) \frac{\langle a\rangle^{2}}{a_{2}^{2}} .
$$

Equation (23) indicates the dependence of the permeability on the specific surface area expressed by square of the area-averaged radius. By means of Eq. (8), Eq. (23) for the same mass fraction $\left(x_{1}=x_{2}=0.5\right)$ is obtained as $k^{*}=k_{\text {mono }}^{*}(\phi)[2 R /(1+R)]^{2}$. Figure 7 indicates that Eq. (23) could roughly represent the permeability of uniform particulate bed even though there are slight differences from our results and those by van der Hoef et al. (2005).

For intermediate cases, the particulate bed does not consist of definite layers and therefore the particle size distribution varies with position. By using Eqs. (10) and (11), which express the permeability by adding the permeation resistance of individual layer, the total permeability of locally-stratified particulate bed can be calculated as

$$
k^{*}=\left[\int_{-L^{*} / 2}^{L^{*} / 2} \frac{1}{L^{*}} \frac{1}{k\left(z^{*}\right) / a_{2}^{2}} d z^{*}\right]^{-1}=\left[\int_{-L^{*} / 2}^{L^{*} / 2} \frac{1}{L^{*}} \frac{1}{k_{\text {mono }}^{*}(\phi)\left\langle a\left(z^{*}\right)\right\rangle^{2} / a_{2}^{2}} d z^{*}\right]^{-1},
$$

where $k\left(z^{*}\right) / a_{2}^{2}$ is dimensionless local permeability at tiny regions within a bed and $\left\langle a\left(z^{*}\right)\right\rangle$ is local area-averaged radius which is determined by size and spatial distribution of the particles. The right-hand side in Eq. (24) is obtained from the dependence of the permeability on the specific surface area on the basis of Eq. (23). If the particle volume fraction is constant in the bed, the total permeability is obtained as the product of the permeability of monodisperse bed and the correction term concerning the particle size distribution as follows.

$$
k^{*}=k_{\mathrm{mono}}^{*}(\phi)\left[\frac{a_{2}^{2}}{L^{*}} \int_{-L^{*} / 2}^{L^{*} / 2} \frac{d z^{*}}{\left\langle a\left(z^{*}\right)\right\rangle^{2}}\right]^{-1} .
$$

In our calculation, the integral in Eq. (25) can be calculated from the existing probabilities $g_{1}$ $\left(z^{*}\right)$ and $g_{2}\left(z^{*}\right)$ which include the stratification degree $\lambda$ and the radius ratio $R$. We omit the lengthy derivation here and only show the results in Fig.7. It is found that Eq. (25) approaches to Eq. (22) for small $\lambda$ and approaches to Eq. (23) for large $\lambda$, and it almost reproduces our calculation results for a wide range of $\lambda$ and $R$. There is a deviation from the calculation 
results in the case of $R=5$. This is interpreted as an error in the calculation results which arises from a sprinkling of larger particles and it might decrease to some extent by larger-scale computation. The results indicate that the total permeability of heterogeneous bidisperse bed can be approximately described by integrating the local permeation resistance which depends on the local specific surface area of the constituent particles.

The results shown in Fig.7 are useful for a practical perspective since the stratification degree $\lambda$ roughly express the actual lenghscale of mixing region of bidisperse particulate bed. If one wants to know the permeability of the partially-mixed bidisperse bed, e.g., sand and gravel layers with their mixing region, it can be roughly estimated from Fig.7 with $\lambda$ (lenghscale of mixing region) and $R$ (radius ratio).

\subsection{Permeability in stratified bed of polydisperse particles}

The structural effect in polydisperse particulate bed on the permeation characteristics is also examined. The particle size in the bed is given by the log-normal distribution which is commonly employed as size distribution of granular particles. When the diameter of particle $l$ is represented by $D_{l}$, the log-normal distribution function is described by

$$
f\left(D_{l}\right)=\frac{1}{D_{l} \ln \sigma \sqrt{2 \pi}} \exp \left[-\frac{\left(\ln D_{l}-\ln D_{50}\right)^{2}}{2(\ln \sigma)^{2}}\right],
$$

where $D_{50}$ is the median particle diameter and $\sigma$ is the standard deviation. The greater $\sigma$ represents the particle size distribution with higher polydispersity. We consider the particulate beds for $\ln \sigma=0.2$ and 0.4 . The appearance and particle size distribution of these particulate beds are shown in Fig. 8. The particle radius $a_{l}$ is equal to half of $D_{l}$ and the normalized radius $a_{l} / a_{0}$ ranges from 0.6 to 2.2 for $\ln \sigma=0.2$ and from 0.2 to 4.0 for $\ln \sigma=0.4$. The representative radius $a_{0}$ is set to be $D_{50} / 2$.

Firstly we show the results for three-dimensionally infinite systems. Figure 9 indicates the calculation results of the total permeability $k^{*}$ in uniform (well-mixed) polydisperse bed. The expression of the permeability for polydisperse system has been proposed by Sarkar et al. (2009). It is found that our results have the same tendency as those by Sarkar et al. (2009) and particularly both results agree for small $\phi$. 
In order to investigate the internal stratification effects on permeability for polydisperse systems, we also calculate the permeability of locally-stratified polydisperse bed with finite thickness. We arranged the particles in the bed according to the following probability function.

$$
h_{l}\left(z^{*}\right)=\frac{1}{2}\left[\operatorname{erf}\left\{-\frac{1}{\lambda}\left(z^{*}-q_{l}\right)\right\}+1\right],
$$

where $h_{l}$ indicates the volumetric existing probability of particles which is larger than particle l. $z^{*}\left(=z / a_{0}\right)$ is the normalized position and the particulate bed locates from $z^{*}=0$ to $L^{*} \cdot \lambda$ is the stratification degree and $q_{l}$ is the arbitrary constant. $q_{l}$ is determined in order that the local particle volume fraction does not vary within the overall bed. By means of Eq. (27), the volumetric existing probability of particle $l$ is calculated as $g_{l}\left(z^{*}\right)=h_{l}\left(z^{*}\right)-h_{l-1}\left(z^{*}\right)$. The common form of the probability density function can be written as $p_{l}\left(z^{*}\right)=g_{l}\left(z^{*}\right) /\left[a_{0} \int_{0}^{L^{*}} g_{l}\left(z^{*}\right) d z^{*}\right]$. Figure 10 indicates the size and spatial distributions of particles calculated from Eq. (27) with different stratification degree $\lambda$ for $\ln \sigma=0.4$. Figure 11 is the illustration of the corresponding particulate beds to Fig. 10 for $\phi=0.2$. As shown in Figs. 10 and 11 , the parameter $\lambda$ plays a similar role as that in the previous section. In other words, the bed is multilayered for small $\lambda$ while it is uniform (well-mixed) for large $\lambda$.

Figure 12 shows the calculation results of permeability profile of the particulate beds illustrated in Fig. 11. It is found that the permeability is greatly influenced by the spatial distribution of different-sized particles. For $\lambda=200$, the permeability at most of the position indicates the same value as that of the infinite particulate bed which is shown in Fig.9 (represented by dotted line in Fig. 12). On the other hand, the permeability for $\lambda=2$ varies with position and it drastically increases at the region in which the larger particles are concentrated. The end effect at such a region is more definite owing to its dependence on the particle size as shown in Fig. 3. As a result, the local permeability of polydisperse particulate bed is extremely sensitive to the size and spatial distributions of constituent particles.

Figure 13 shows the relation between the stratification degree $\lambda$ and the dimensionless permeability $k^{*}$. As is the case with bidisperse particulate systems (see Fig.7), the permeability is dependent on the stratification degree $\lambda$ and the dependence is stronger if the polydispersity 
is higher. The lower limit of $\lambda$ corresponds to multilayered (completely-stratified) system. In this case, the permeability can be calculated from Eq. (11) which represents the additivity of the permeation resistance of the constituent monodisperse layers. On the other hand, the higher limit of $\lambda$ indicates completely-mixed system and the permeability can be calculated from Eq. (23), which represents the relation between the permeability and the specific surface area. It is found from Fig. 13 that both approximated solutions are almost in agreement with calculation results for small and large $\lambda$.

For intermediate cases, the permeability can be calculated from the integration of the local permeation resistance as follows:

$$
k^{*}=\left[\int_{0}^{L^{*}} \frac{1}{L^{*}} \frac{1}{k_{\mathrm{mono}}^{*}(\phi)\left\langle a\left(z^{*}\right)\right\rangle^{2} / a_{0}^{2}} d z^{*}\right]^{-1}=k_{\mathrm{mono}}^{*}(\phi)\left[\frac{a_{0}^{2}}{L^{*}} \int_{0}^{L^{*}} \frac{d z^{*}}{\left\langle a\left(z^{*}\right)\right\rangle^{2}}\right]^{-1} .
$$

Equation (28) is derived in a similar way to bidisperse system indicated in Eqs. (24) and (25), i.e., the local permeability is described as a function of the local specific surface area of the particles. Figure 13 shows that Eq. (28) could roughly describe the permeability of partiallymixed particulate bed. These results suggest that the total permeability of heterogeneous particulate media can be estimated by integration of the local permeation resistance which is determined by the specific surface area of polydisperse particles at each location.

\section{Conclusions}

The permeability of unconsolidated polydisperse particulate bed with finite thickness is calculated by means of Stokesian dynamics approach. From relation between permeability of random particulate bed and particle volume fraction, we have confirmed that the results by our calculation method denote the same tendency of those shown in previous works and both results agree for small particle volume fraction. In order to investigate the permeation characteristics of non-uniform particulate bed, we define the stratification degree as a parameter, which apparently and mathematically corresponds to the thickness of the mixing region of different-sized particles. The results for different stratification degree suggest that the permeability greatly depends on the spatial distribution of particles with different radius. 
Consequently, it is found that the permeability of non-uniform particulate media can be obtained from integration of the local permeation resistance determined by specific surface area of the polydisperse particles.

This work was supported by Grant-in-Aid for JSPS Fellows $23 \cdot 4066$.

\section{REFERENCES}

Beenakker, C. W. J.: Ewald sum of the Rotne-Prager tensor. J. Chem. Phys. 85(3), 1581-1582 (1986).

Brady, J. F., Phillips, R. J., Lester, J. C., Bossis, G.: Dynamic simulation of hydrodynamically interacting suspensions. J. Fluid Mech. 195, 257-280 (1988).

Brinkman, H. C.: On the permeability of media consisting of closely packed porous particles. Appl. Sci. Res. A1, 81-86 (1947).

Carman, P. C.: Fluid flow through granular beds. Trans. Inst. Chem. Eng. 15, 150-166 (1937).

Cello, F., Di Renzo, A., Di Maio, F. P.: A semi-empirical model for the drag force and fluid-particle interaction in polydisperse suspensions. Chem. Eng. Sci. 65, 3128-3139 (2010).

Clague, D. S., Phillips, R. J.: A numerical calculation of the hydraulic permeability of threedimensional disordered fibrous media. Phys. Fluids 9(6), 1562-1572 (1997).

Drummond, J. E., Tahir, M. I.: Laminar viscous flow through regular arrays of parallel solid cylinders. Int. J. Multiphase Flow 10(5), 515-540 (1984).

Durlofsky, L., Brady, J. F., Bossis, G.: Dynamic simulation of hydrodynamically interacting particles. J. Fluid Mech. 180, 21-49 (1987).

Happel, J.: Viscous flow in multiparticle systems: Slow motion of fluids relative to beds of spherical particles. A.I.Ch.E. J. 4(2), 197-201 (1958).

Happel, J., Brenner, H.: Low Reynolds number Hydrodynamics, Kluwer Academic Publishers, Dordrecht (1991).

Harada, S., Otomo, R.: Diffusive behavior of a thin particle layer in fluid by hydrodynamic interaction. Phys. Rev. E (2009). doi:10.1103/PhysRevE.80.066311

Hill, R. J., Koch, D. L., Ladd, A. J. C.: The first effects of fluid inertia on flows in ordered and random arrays of spheres. J. Fluid Mech. 448, 213-241 (2001a).

Hill, R. J., Koch, D. L., Ladd, A. J. C.: Moderate-Raynolds-number flows in ordered and random arrays of spheres. J. Fluid Mech. 448, 243-278 (2001b).

Hirono, T., Takahashi, M., Nakashima, S.: In situ visualization of fluid flow image within deformed rock by X-ray CT. Eng. Geol. 70, 37-46 (2003).

Ichiki, K., Master Thesis, Tohoku University (1993).

Innocentini, M. D. M., Rodrigues, V. P., Romano, R. C. O., Pileggi, R. G., Silva, G. M. C., Coury, J. R.: Permeability optimization and performance evaluation of hot aerosol filters made using foam incorporated alumina suspension. J. Hazard. Mater. 162, 212-221 (2009).

Ishii, K.: Viscous flow past multiple planar arrays of small spheres. J. Phys. Soc. Jpn. 46(2), 675680 (1979).

Jackson, G. W., James, D. F.: The permeability of fibrous porous media. Can. J. Chem. Eng. 64, 364-374 (1986).

Jeffrey, D. J., Onishi, Y.: Calculation of the resistance and mobility functions for two unequal rigid spheres in low-Reynolds-number flow. J. Fluid Mech. 139, 261-290 (1984).

Kim, S., Karrila, S. J.: Microhydrodynamics, Butterworth-Heinemann, Boston and London (1991).

Koo, S., Sangani, A. S.: Effective-medium theories for predicting hydrodynamic transport properties of bidisperse suspensions. Phys. Fluids 14(10), 3522-3533 (2002).

Ladd, A. J. C.: Hydrodynamic transport coefficients of random dispersions of hard spheres. J. Chem. Phys. 93(5), 3484-3494 (1990).

Liekens, A., Billen, J., Sherant, R., Ritchie, H., Denayer, J., Desmet, G.: High performance liquid chromatography column packings with deliberately broadened particle size distribution: Relation between column performance and packing structure. J. Chromatogr. A 1218, 6654-6662 (2011). Otomo, R., Harada, S.: End effect on permeability of loose particulate bed having different internal 
structures. Particul. Sci. Technol. 29, 1-12 (2011).

Rotne, J., Prager, S.: Variational treatment of hydrodynamic interaction in polymers. J. Chem. Phys. 50(11), 4831-4837 (1969).

Sangani, A. S., Acrivos, A.: Slow flow past periodic arrays of cylinders with application to heat transfer. Int. J. Multiphase Flow 8(3), 193-206 (1982).

Sangani, A. S., Behl, S.: The planar singular solutions of Stokes and Laplace equations and their application to transport processes near porous surfaces. Phys. Fluids A 1(1), 21-37 (1989).

Sarkar, S., van der Hoef, M. A., Kuipers, J. A. M.: Fluid-particle interaction from lattice Boltzmann simulations for flow through polydisperse random arrays of spheres. Chem. Eng. Sci. 64, 2683-2691 (2009).

van der Hoef, M. A., Beetstra, R., Kuipers, J. A. M.: Lattice-Boltzmann simulations of lowReynolds-number flow past mono- and bidisperse arrays of spheres: results for the permeability and drag force. J. Fluid Mech. 528, 233-254 (2005).

Vidal, D., Ridgway, C., Pianet, G., Schoelkopf, J., Roy, R., Bertrand, F.: Effect of particle size distribution and packing compression on fluid permeability as predicted by lattice-Boltzmann simulations. Comput. Chem. Eng. 33, 256-266 (2009). 


\section{Figure captions}

Fig. 1 Schematic diagram of particulate beds with layered, uniform, and intermediate structures.

Fig. 2 Dimensionless permeability $k^{*}$ of infinite and uniform particulate bed for various particle volume fraction $\phi$.

Fig. 3 Permeability profiles in finite particulate beds $(\phi=0.2)$. The results are normalized by the permeability of infinite monodisperse bed $k_{\text {mono }}^{*}\left(\phi=0.2, a / a_{0}=1.0\right)$, where $a$ represents the radius of monodisperse particulate bed. Straight lines are the permeability of infinite particulate bed: Dotted line and broken line represent monodisperse bed with $a / a_{0}=1.0$ and $a / a_{0}=2.0$, respectively. Dotted-broken line represents bidisperse bed with $R=2$. The bed thickness is $L^{*}=45$.

Fig. 4 Probability profile of larger particle in $z$ direction with different stratification degree $\lambda$.

Fig. 5 Illustration of particulate bed with different stratification degree $\lambda(\phi=0.2, R=3)$.

Fig. 6 Permeability profiles in particulate bed with different stratification degree $\lambda(\phi=0.2, R=3)$. The results are normalized by the smaller particle radius $a_{2}$. Straight lines are the permeability of infinite particulate bed with uniform structure at the same particle volume fraction $(\phi=0.2)$ : Dotted line and broken line represent monodisperse particulate bed with $a / a_{2}=1.0$ and $a / a_{2}=3.0$, respectively. Dotted-broken line represents bidisperse particulate bed with $R=3$.

Fig. 7 Relation between stratification degree $\lambda$ and total permeability $k^{*}$. The results are normalized by the permeability of infinite monodisperse bed $k_{\text {mono }}^{*}(\phi=0.2)$. The numbers of each particle are set so that the mass fraction satisfies $x_{1}=x_{2}=0.5$ in all cases of radius ratio: $N_{1}=555$ and $N_{2}=4441$ for $R=2, N_{1}=178$ and $N_{2}=4807$ for $R=3$, and $N_{1}=39$ and $N_{2}=4876$ for $R=5$.

Fig. 8 Appearance of the polydisperse particulate beds $(\phi=0.2)$ and particle size distribution. Total number of particles is $N=5000$ in both cases.

Fig. 9 Dimensionless permeability of polydisperse particulate bed $k^{*}$ for various particle volume fractions $\phi$. The bed is three-dimensionally infinite and internal particle arrangement is uniform (well-mixed).

Fig. 10 Volumetric existing probability of particle larger than particle $l$ with different stratification degree $\lambda(\ln \sigma=0.4)$. The legends of (b) and (c) are the same as (a).

Fig. 11 Illustration of the particulate beds with different stratification degree $\lambda(\phi=0.2, \ln \sigma=0.4)$.

Fig. 12 Permeability profile in particulate bed with different stratification degree $\lambda(\phi=0.2, \ln \sigma$ $=0.4$ ). Dotted line represents the results for infinite and uniform particulate bed with the same particle size distribution.

Fig. 13 Relation between stratification degree $\lambda$ and dimensionless total permeability $k^{*}(\phi=0.2)$. 
(a)

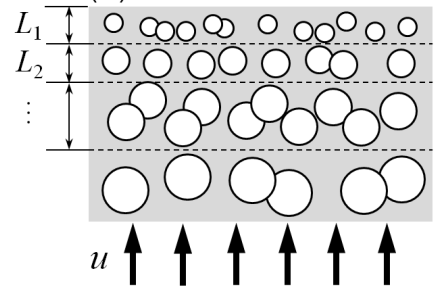

layered structure (b)

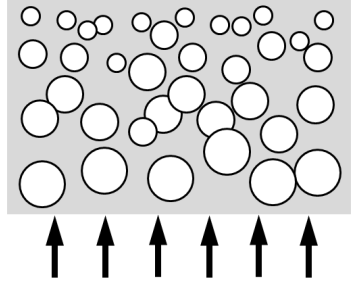

(c)

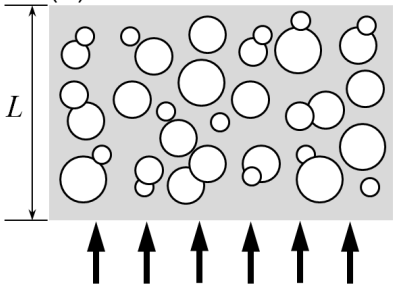

uniform structure

Fig. 1

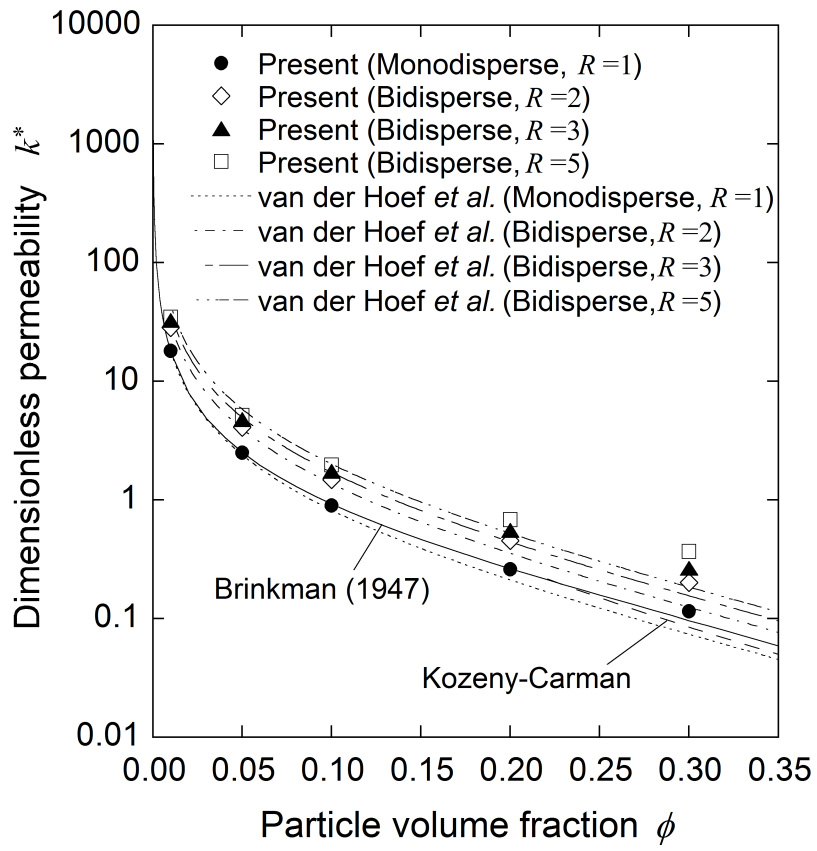

Fig. 2 


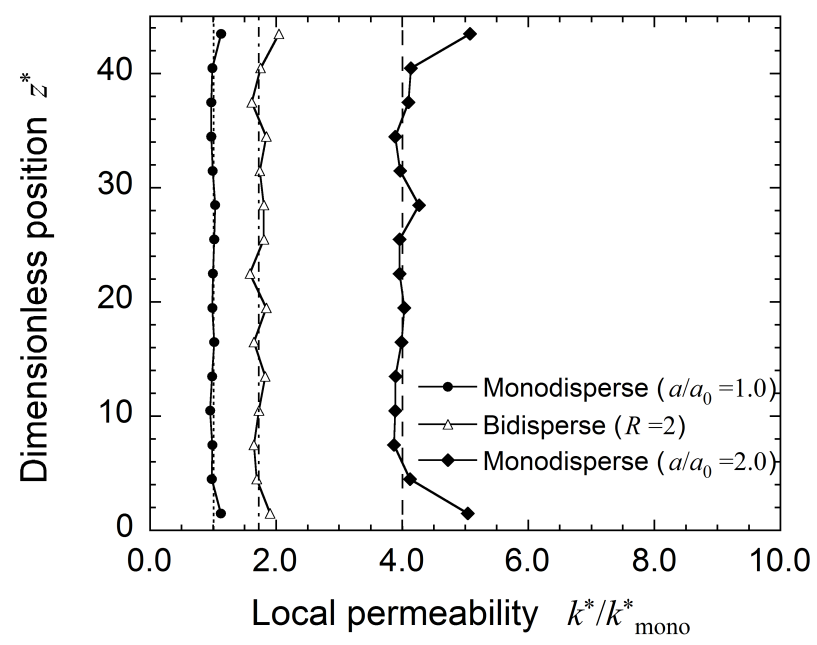

Fig. 3

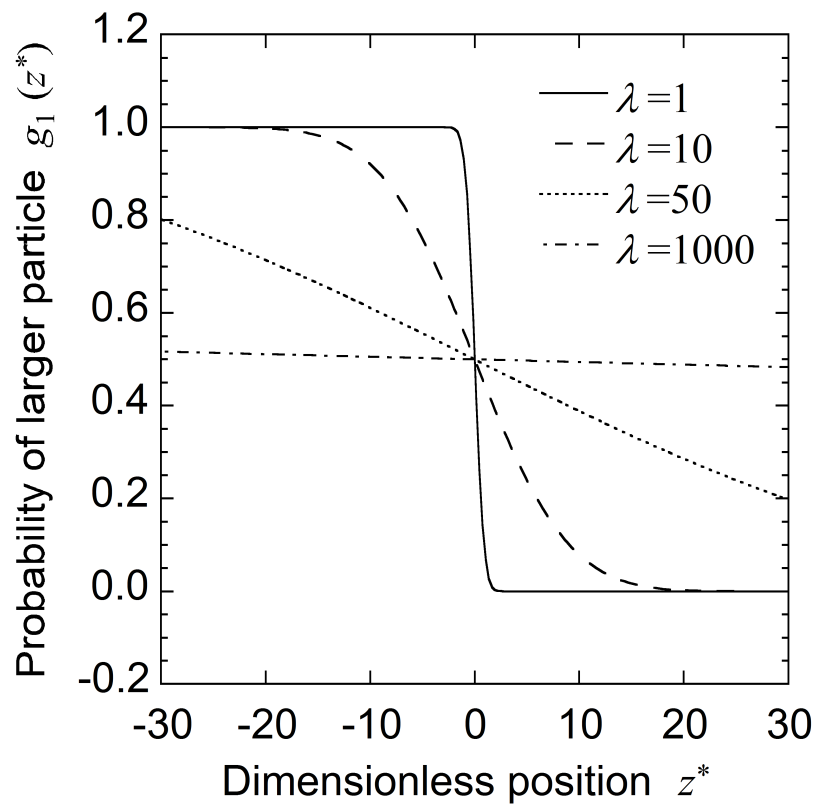

Fig. 4 


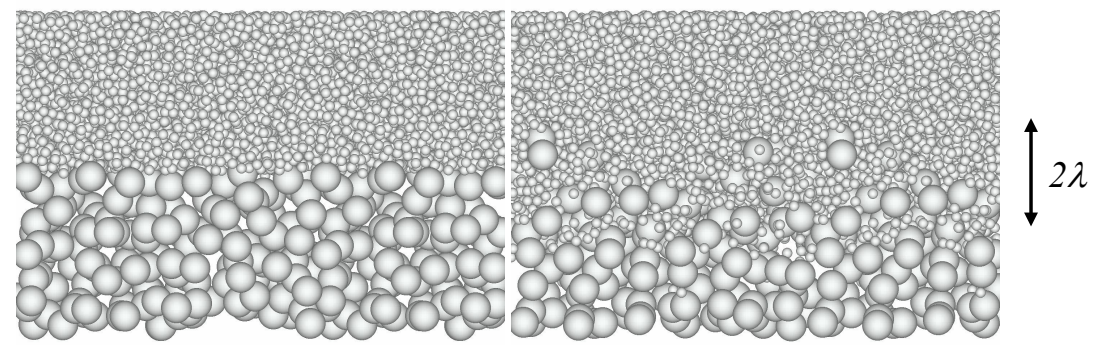

(a) $\lambda=1$

(b) $\lambda=10$

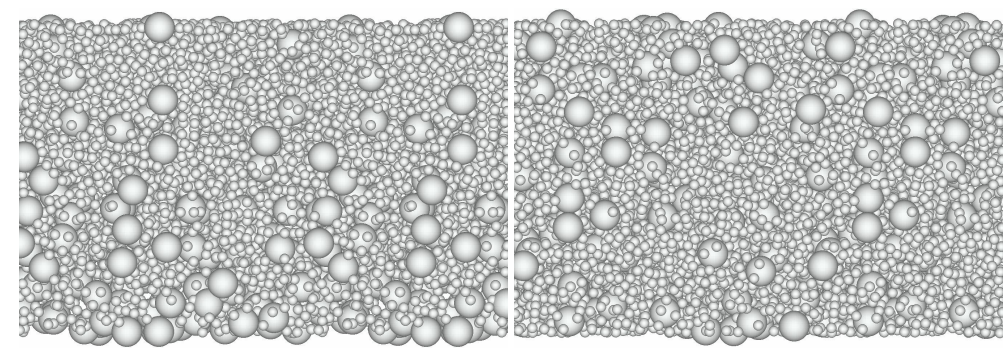

(c) $\lambda=50$

(d) $\lambda=1000$

Fig. 5

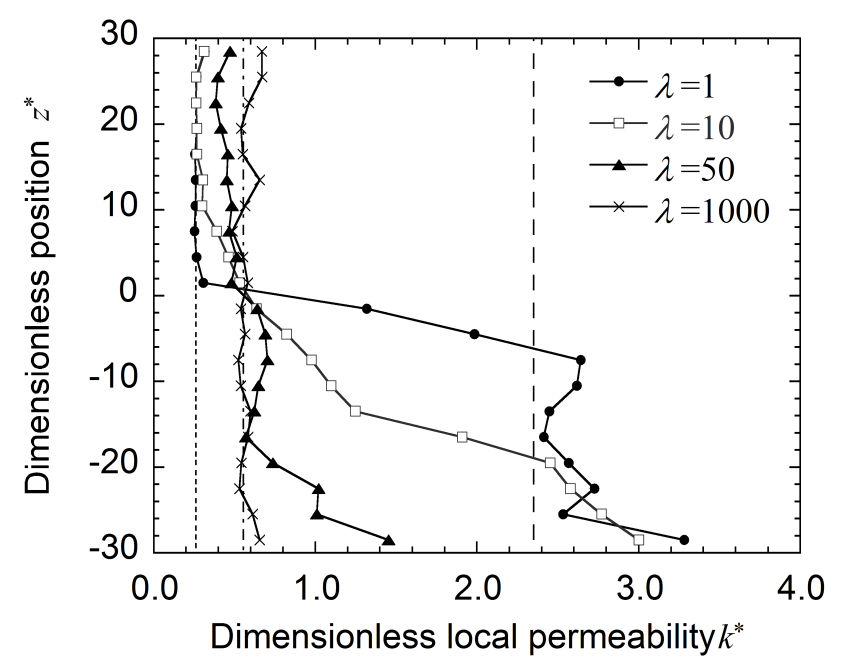

Fig. 6 


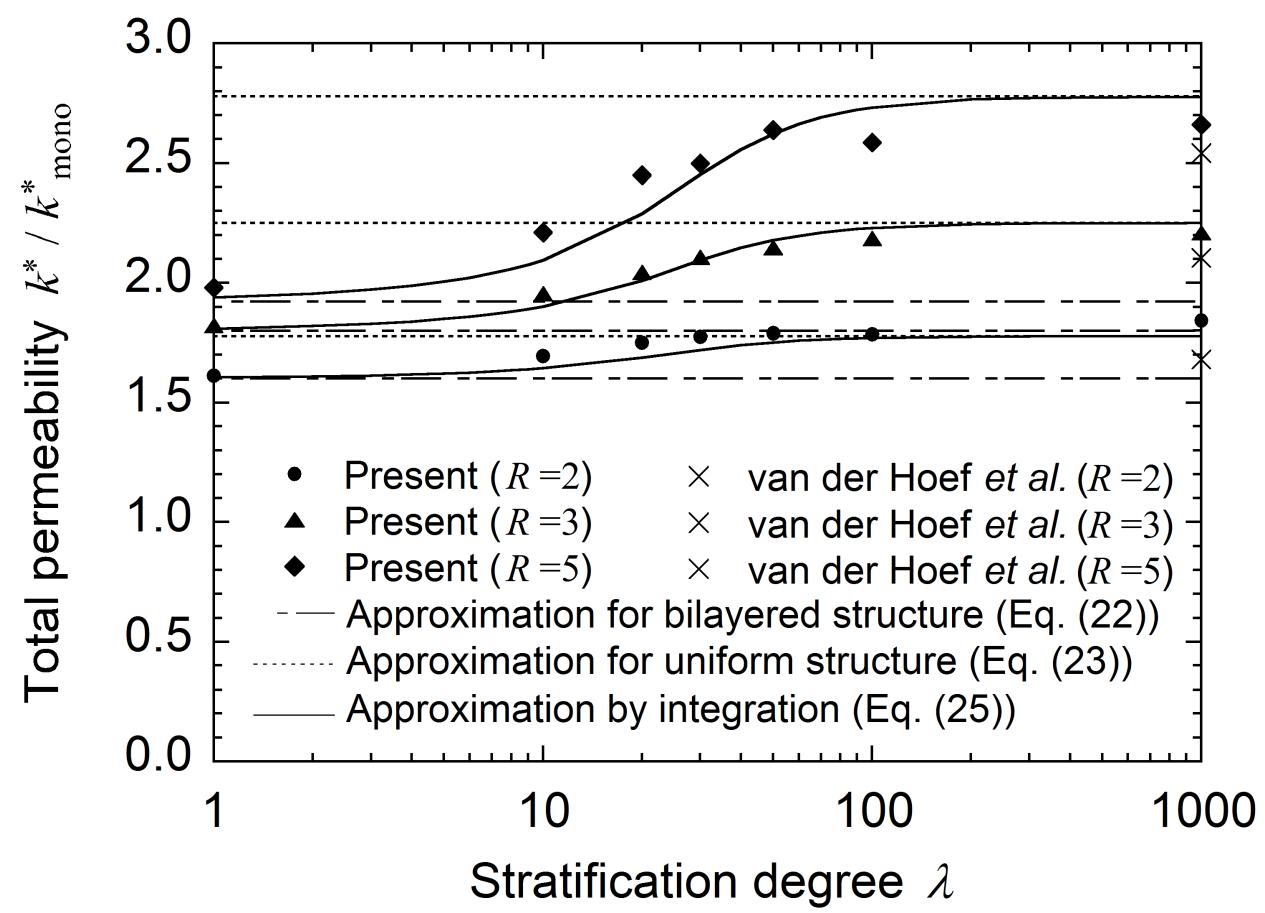

Fig. 7 

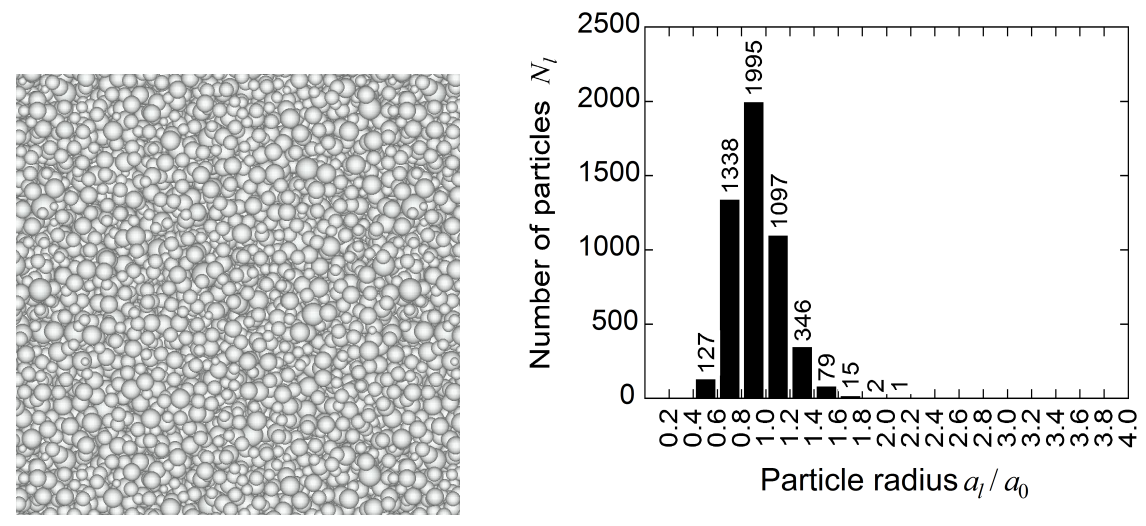

(a) $\ln \sigma=0.2$
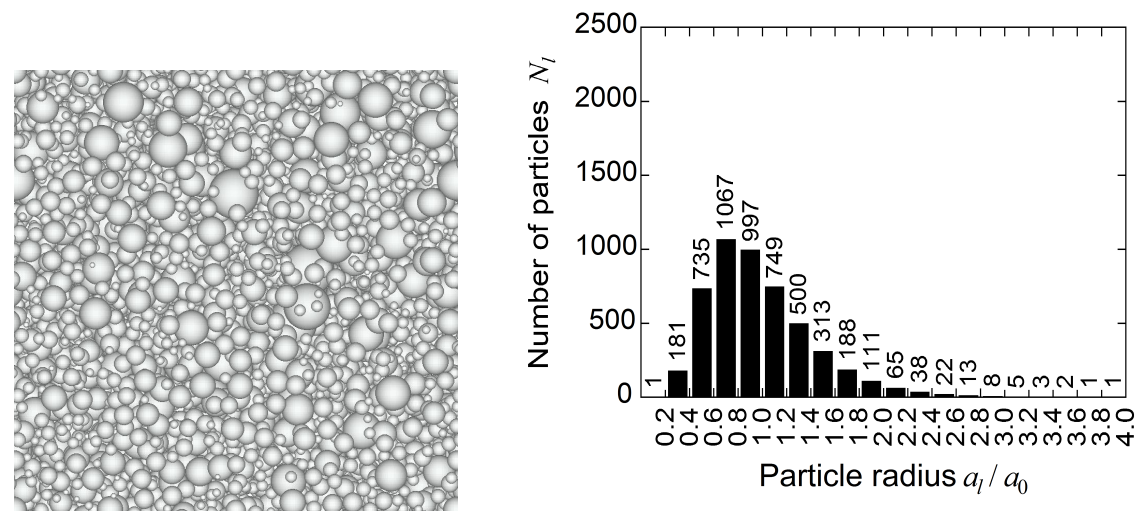

(b) $\ln \sigma=0.4$

Fig. 8

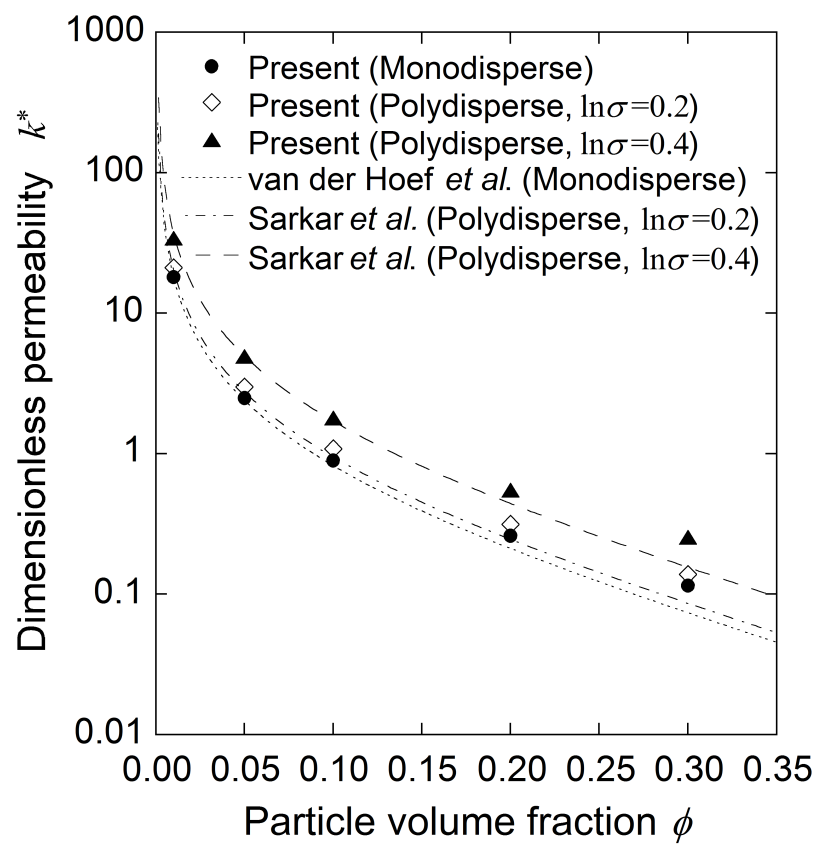

Fig. 9 


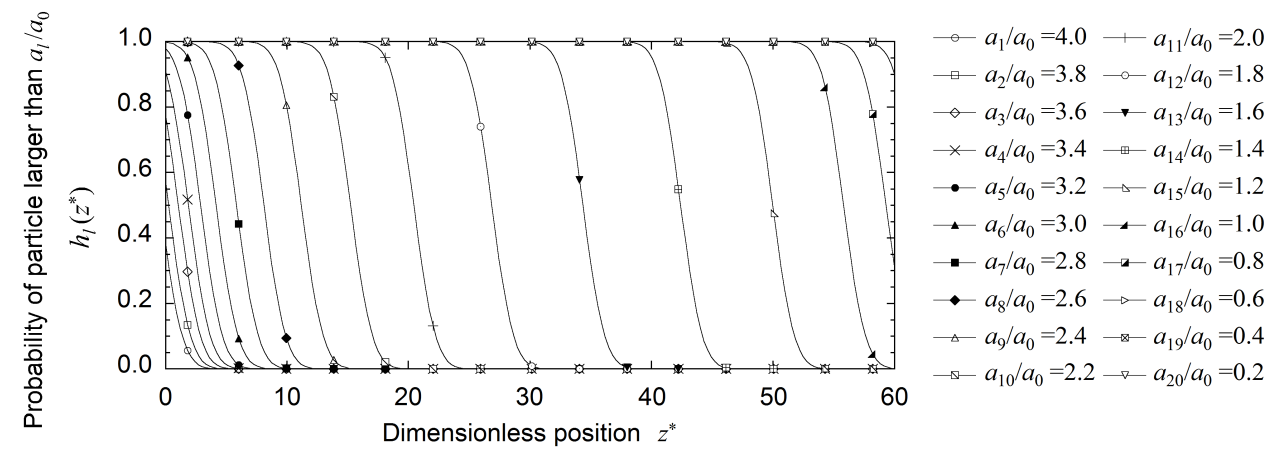

(a) $\lambda=2$

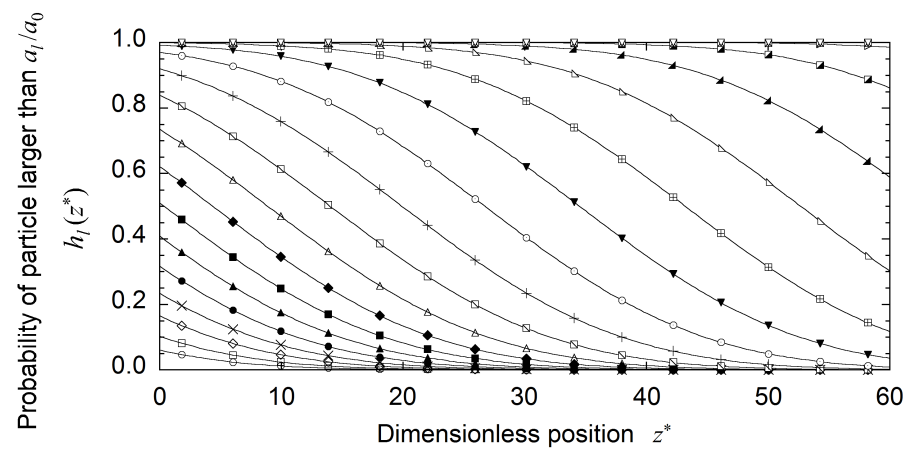

(b) $\lambda=20$

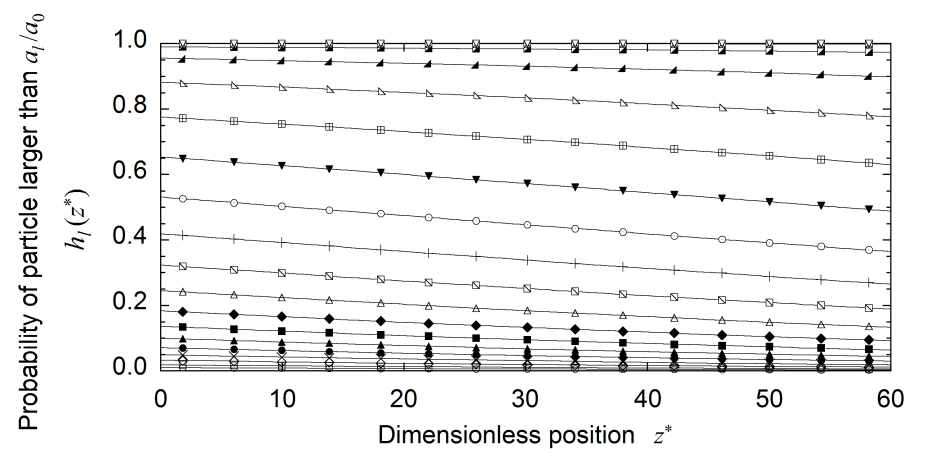

(c) $\lambda=200$

Fig. 10 


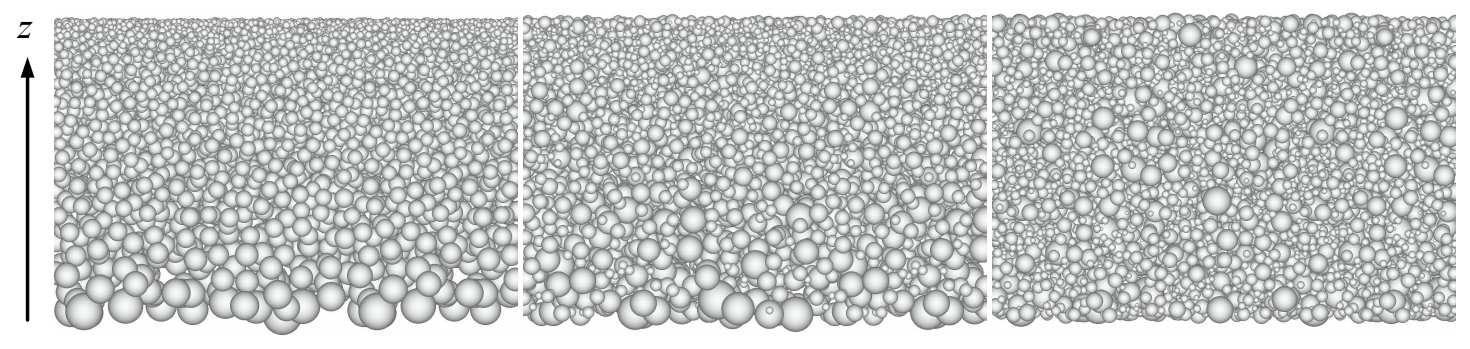
(a) $\lambda=2$
(b) $\lambda=20$
(c) $\lambda=200$

Fig. 11

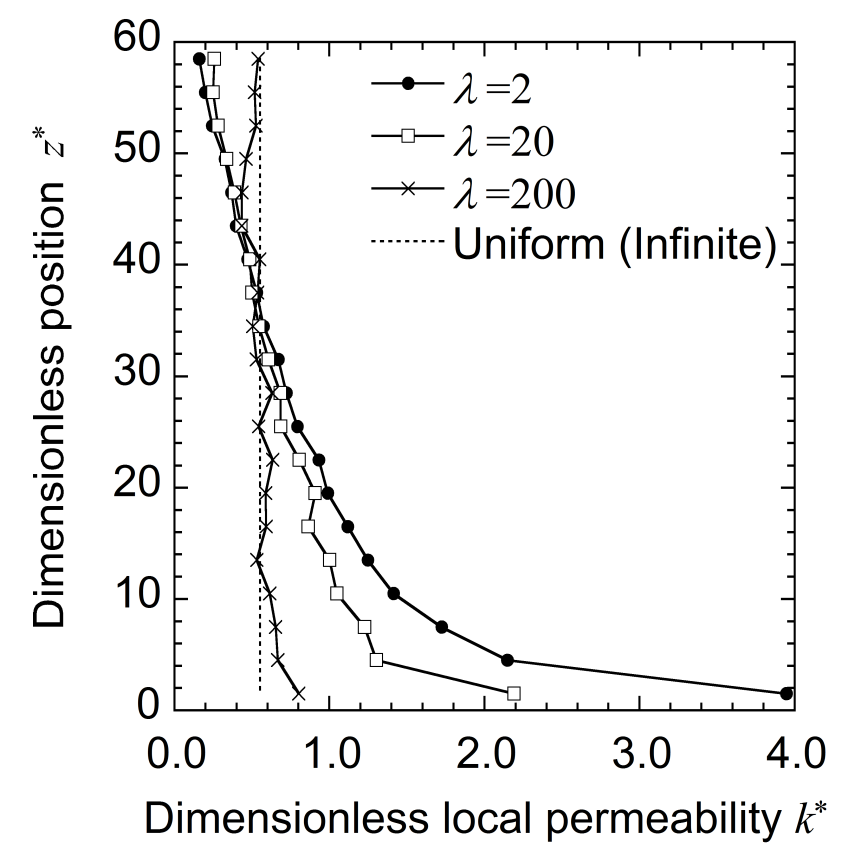

Fig. 12 


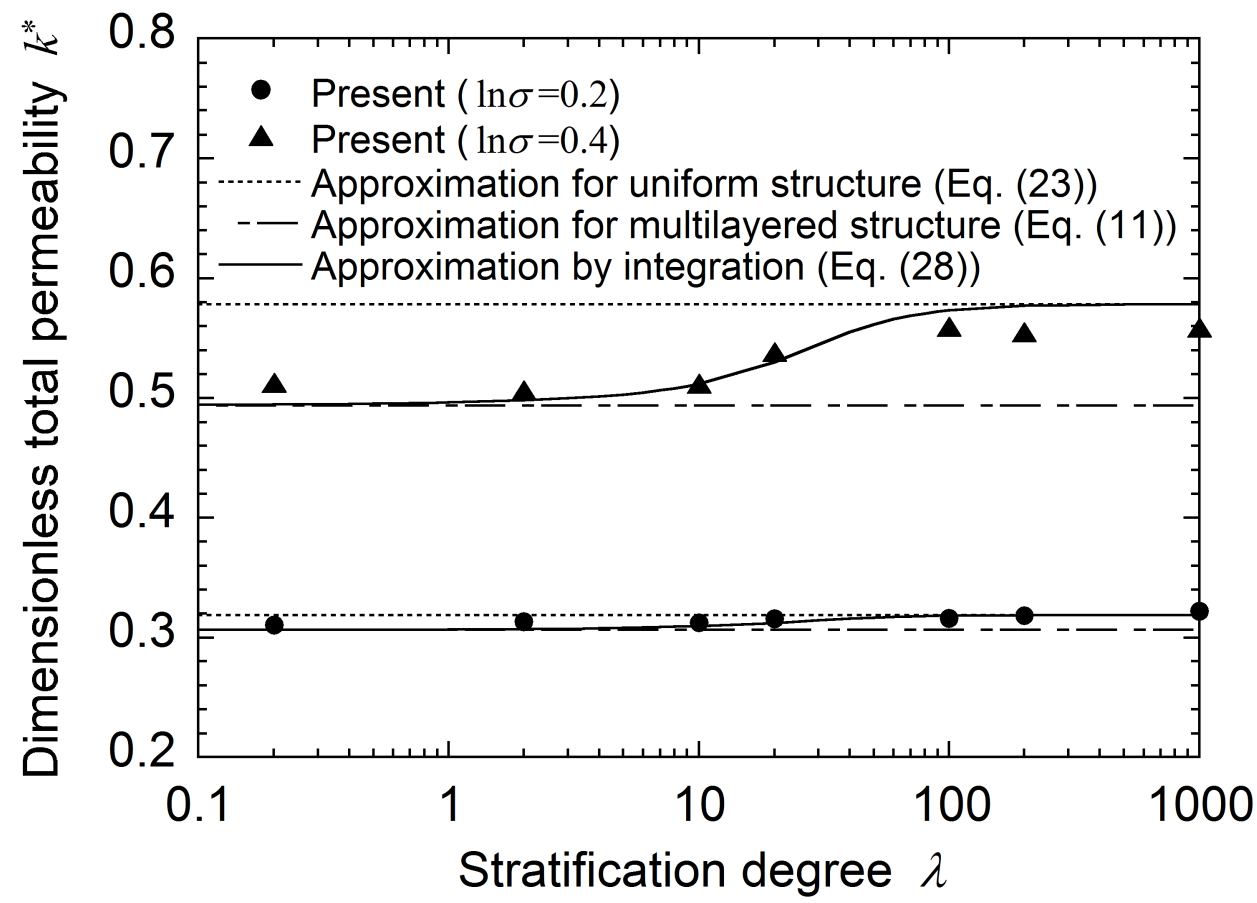

Fig. 13 